\title{
Parallel Midbrain Microcircuits Perform Independent Temporal Transformations
}

\author{
C. Alex Goddard, ${ }^{1}$ John Huguenard, ${ }^{2}$ and Eric Knudsen ${ }^{1}$ \\ ${ }^{1}$ Department of Neurobiology, Stanford University, Stanford, California 94305, and 2Department of Neurology, Stanford Medical School, Stanford, \\ California 94305
}

The capacity to select the most important information and suppress distracting information is crucial for survival. The midbrain contains a network critical for the selection of the strongest stimulus for gaze and attention. In avians, the optic tectum (OT; called the superior colliculus in mammals) and the GABAergic nucleus isthmi pars magnocellularis (Imc) cooperate in the selection process. In the chicken, OT layer 10, located in intermediate layers, responds to afferent input with gamma periodicity $(25-75 \mathrm{~Hz})$, measured at the level of individual neurons and the local field potential. In contrast, Imc neurons, which receive excitatory input from layer 10 neurons, respond with tonic, unusually high discharge rates ( $>150$ spikes/s). In this study, we reveal the source of this high-rate inhibitory activity: layer 10 neurons that project to the Imc possess specialized biophysical properties that enable them to transform afferent drive into high firing rates $(\sim 130$ spikes/s), whereas neighboring layer 10 neurons, which project elsewhere, transform afferent drive into lower-frequency, periodic discharge patterns. Thus, the intermediate layers of the OT contain parallel, intercalated microcircuits that generate different temporal patterns of activity linked to the functions of their respective downstream targets.

Key words: attention; colliculus; decision; inhibition; tectum

\section{Introduction}

Animals must select the most important stimuli to guide behavior. The study of attention suggests that the selection process involves two components: (1) enhancement of the selected representations; and (2) suppression of the nonselected representations. The enhancement of attended representations involves top-down connectivity from frontal forebrain regions (Armstrong et al., 2006; Miller and Buschman, 2013) and neuromodulators, such as acetylcholine (Herrero et al., 2008). However, the suppression of distracting, nonselected representations has been less well studied. This suppressive component is crucial: human studies indicate that reliable detection of a stimulus is dependent on active suppression of irrelevant stimuli (Gazzaley et al., 2005). The mechanisms of suppression are not well known, although they likely involve the action of potent GABAergic circuitry.

The midbrain contains a network crucial for the selection of salient stimuli (Lovejoy and Krauzlis, 2010; Knudsen, 2011). In the avian midbrain, the optic tectum (OT) and the GABAergic

Received Oct. 15, 2013; revised May 3, 2014; accepted May 5, 2014.

Author contributions: C.A.G., J.H., and E.K. designed research; C.A.G. performed research; C.A.G. contributed unpublished reagents/analytic tools; C.A.G. analyzed data; C.A.G., J.H., and E.K. wrote the paper.

This work was supported by a Stanford Dean's Postdoctoral fellowship (C.A.G.), National Eye Institute Grants F32 EY018787 (C.A.G.) and EY019179-33 (E.K.), and National Institute of Neurological Disorders and Stroke Grant NS34774 (J.H.). Special thanks to Stefan Heller, Shreesh Mysore, Astra Bryant, Sridhar Devarajan, Ali Asadollahi, Jason Schwarz, Koji Takahashi, and members of the Knudsen and Huguenard laboratories for assistance and useful discussions.

The authors declare no competing financial interests.

Correspondence should be addressed to C. Alex Goddard, 299 Campus Drive, Stanford, CA 94305. E-mail: cgoddard@stanford.edu.

DOI:10.1523/JNEUROSCI.4399-13.2014

Copyright $\odot 2014$ the authors $\quad 0270-6474 / 14 / 348130-09 \$ 15.00 / 0$ nucleus isthmi pars magnocellularis (Imc) interact to select the strongest stimulus (Fig. 1A; Mysore and Knudsen, 2013). The Imc receives topographic input from the OT and sends a broad inhibitory projection to the OT to implement competitive suppression (Wang et al., 2004; Marín et al., 2007; Mysore and Knudsen, 2013). Imc neurons respond to stimuli with high spike rates (Marín et al., 2007), thus providing a continuous stream of the inhibitory neurotransmitter GABA to its postsynaptic targets. Therefore, the OT-Imc circuit is a model for studying the mechanisms of stimulus selection.

A perplexing puzzle arises concerning how the exceptionally high firing rates in the Imc are generated. The Imc receives input from intermediate OT layer 10 (L10) neurons (Fig. 1B; Wang et al., 2004), many of which fire at rates in the low gamma band (25-75 spikes/s) in vitro (Goddard et al., 2012) and coherently with a gamma band local field potential (LFP) in vivo (Sridharan et al., 2011). Furthermore, L10 neurons provide periodic, gamma-band inputs to neurons in the nearby cholinergic nucleus isthmi pars parvocellularis (Ipc; Goddard et al., 2012).

Given that L10 is dominated by neurons that discharge at low rates, how do Imc neurons generate the high firing rates necessary for their function? In this study, we propose and test three hypotheses (Fig. 1C): (1) biophysical properties of Imc neurons integrate and transform low-rate inputs from L10 into high rates of spiking; (2) Imc-projecting L10 neurons fire at low rates but are temporally jittered relative to each other, providing high-rate inputs to the Imc; or (3) Imc-projecting L10 neurons are specialized to fire at high rates, thus providing high-rate inputs to the Imc.

Here, we find that Imc neurons do not integrate their inputs over time but rather respond to barrages of rapid afferent 
A

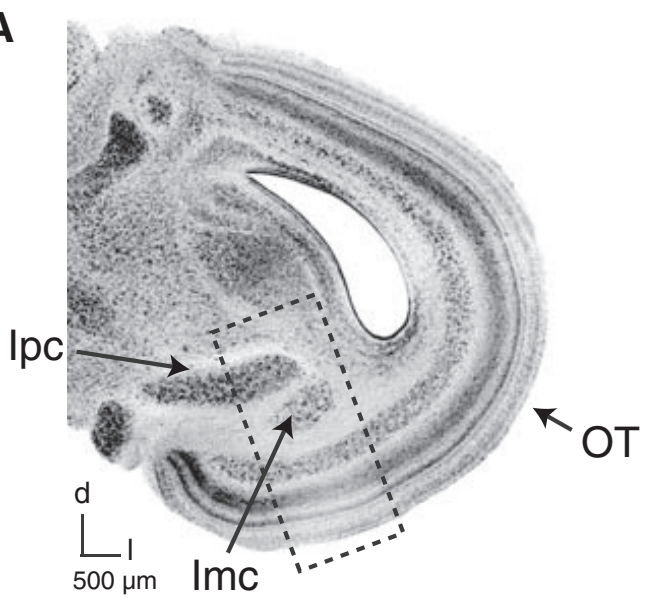

B

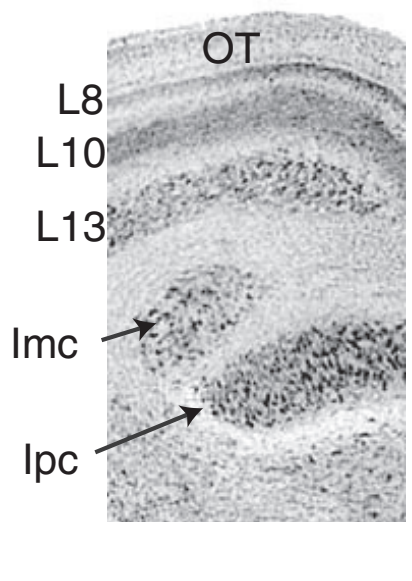

C 1. Imc
Integration

OT
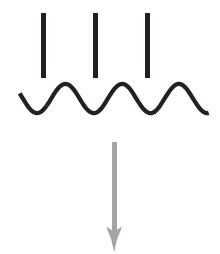

Imc

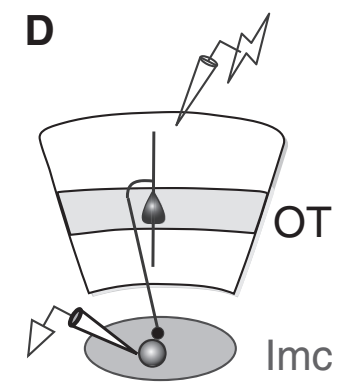

E

2. Low-rate, jittered L10 firing
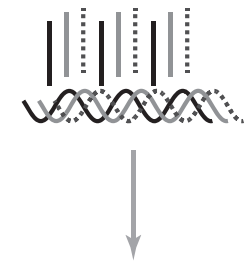

$\||||||| \mid$

Figure 1. Connectivity and activity of the midbrain selection network in vitro. $\boldsymbol{A}$, Transverse Nissl-stained section through the midbrain selection network. The 0T makes reciprocal connections with the Imc and Ipc. Box magnified in B. B, Higher-power view of boxed region in $\boldsymbol{A}$. Neurons in $\mathrm{L} 10$ send excitatory projections to both the Imc and Ipc. $C$, Three hypotheses to explain high rates of spiking activity in the Imc. Left, The intrinsic properties of Imc neurons permit the integration of the inputs from slower L10 neurons (25-75 spikes/s) to generate high-rate firing output. Middle, Multiple L 10 neurons that project to the Imc fire at slow rates but are temporally jittered relative to each other. These jittered inputs yield high input rates onto Imc neurons and thus a high-rate firing output. Right, L10 neurons that project to the Imc fire at high rates, yielding high input rates onto Imc neurons and thus a high-rate firing output. $\boldsymbol{D}$, Schematic of connectivity and experimental setup. Retinal afferents were electrically stimulated (electrode), driving activity in L10 neurons, which in turn drive Imc neurons. Activity was assayed with patch-clamp recordings. $E$, Recordings of Imc activity. Left, An example trace of an Imc neuron firing after retinal afferent microstimulation. Right, Summary plot of evoked Imc neuron firing rates. Thick bar represents the median firing rate, and whiskers represent the 25 and $75 \%$ percentiles. Median firing rate (followed by 25 th and 75 th percentiles): 142 spikes/s (126 and 159 spikes/s); $n=19$. d, Dorsal; I, lateral.

drive with rapid firing output. Moreover, we discovered that Imc-projecting OT neurons have specialized properties that enable firing at high rates, whereas neighboring neurons that project elsewhere fire at lower rates. Thus, a crucial temporal transformation of sensory signaling occurs in the OT: parallel microcircuits in L10 generate distinct temporal patterns of activity to enable the computations performed by their respective downstream structures.
3. High-rate
L10 firing
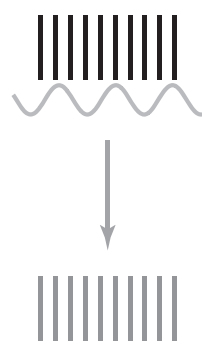

\section{Materials and Methods}

In vitro slice preparation

All animals were treated in accordance with institutional guidelines. White Leghorn and Rhode Island Red chicks (Gallus gallus) of either sex, aged P0-P5, were anesthetized with isoflurane and decapitated, and the brains were removed and immersed in a "cutting" solution $\left(4^{\circ} \mathrm{C}\right)$ containing the following (in $\mathrm{mm}$ ): 234 sucrose, 11 glucose, 24 $\mathrm{NaHCO}_{3}, 2.5 \mathrm{KCl}, 1.25 \mathrm{NaH}_{2} \mathrm{PO}_{4}, 10$ $\mathrm{MgSO}_{4}$, and $0.5 \mathrm{CaCl}_{2}$ (aerated with $95 \% \mathrm{O}_{2}$ and $5 \% \mathrm{CO}_{2}$ ). Tecta were dissected and embedded in low-melting temperature agarose (A2756; Sigma). Transverse slices (300-400 $\mu \mathrm{m}$ ) were cut with a vibrating slicer (Leica VT1200). Slices were incubated in oxygenated artificial CSF (ACSF) containing the following (in mM): $126 \mathrm{NaCl}, 26 \mathrm{NaHCO}_{3}, 1.25$ $\mathrm{NaH}_{2} \mathrm{PO}_{4}, 2 \mathrm{CaCl}_{2}, 1 \mathrm{MgSO}_{4}, 2 \mathrm{KCl}$, and 10 glucose, $\mathrm{pH}$ 7.4. Slices were bathed at $34^{\circ} \mathrm{C}$ for 20-30 min and subsequently at room temperature for a minimum of $30 \mathrm{~min}$ before being transferred to the recording chamber. Slices were selected that contained the Imc, $\mathrm{Ipc}$, and clear lamination in the OT, because these slice have the highest probability of preserving connectivity between the $\mathrm{OT}$ and Imc.

In vitro slice recordings

Whole-cell and cell-attached patch recordings were performed in a submerged chamber with ACSF heated to $32-34^{\circ} \mathrm{C}$. Slices were visualized using a Dodt contrast tube. Pipettes were filled with the following solutions (in $\mathrm{mm}$ ): for Figures 1, 2, 4, 5, $130 \mathrm{~K}$-gluconate, $10 \mathrm{KCl}, 2 \mathrm{NaCl}$, 10 HEPES, 10 EGTA, $4 \mathrm{MgATP}$, and 0.3 NaGTP, pH 7.3 (280-290 mOsm); or for Figure 3, 130 Cs-gluconate, $10 \mathrm{CsCl}, 2 \mathrm{NaCl}, 10$ HEPES, 4 EGTA, 5 QX-314, pH 7.3 (280-290 mOsm). For Imc neuron recordings, thinwalled borosilicate pipettes (Sutter Instruments) with 2-4 M $\Omega$ resistances were used; for OT neuron recordings, thick-walled borosilicate glass pipettes with 5-7 M $\Omega$ resistances were used. Data were amplified using a Multiclamp 700B (Molecular Devices) and digitized by a Digidata 1400 (Molecular Devices) with a sampling rate of $10 \mathrm{kHz}$ and recorded using pClamp 10. Cells with series resistance $<30$ $\mathrm{M} \Omega$ and that did not have resistance fluctuate by $>25 \%$ were used for analysis. Bridge was balanced in current-clamp recordings, but series resistance was not compensated in voltage-clamp recordings. For Cs-gluconate recordings, $V_{\text {holding }}$ was corrected for a -16 $\mathrm{mV}$ junction potential at the time of the recording. For isolating PSCs, the membrane was held at a potential that minimized inward inhibitory currents (approximately $-55 \mathrm{mV}$, corrected for junction potential, just depolarized relative to the calculated reversal potential of $-61 \mathrm{mV}$ ); we could observe justdiscernible outward IPSCs in the voltage-clamp trace.

Retinal afferents were microstimulated with constant-current pulse of 10-250 $\mu \mathrm{A}$, lasting $100 \mu \mathrm{s}$, using theta-glass electrodes (BT-15010; Sutter Instruments), pulled as a patch pipette, and filled with ACSF and chlorided silver wires connected to a stimulus isolator (WPI). 


\section{Antidromically activated imaging-based mapping}

We dissolved $50 \mathrm{mg}$ of Oregon Green-488 BAPTA-1 AM (OGB-1; O-6807; Invitrogen) in a solution of pluronic F-127 (P-6867; Invitrogen), DMSO, and HEPES ACSF (in mM: 10 glucose, $136 \mathrm{NaCl}, 2.5 \mathrm{KCl}$, $1.3 \mathrm{MgCl}_{2}, 10$ HEPES, $2 \mathrm{CaCl}_{2}$ ) for a final concentration of $0.5-1 \mathrm{mM}$ OGB-1, 1-2\% pluronic F-127, and 5-10\% DMSO. After placement of the slice, two to three spots in L10 of the OT, separated by $\sim 200 \mu \mathrm{m}$, were labeled by placing a 1-2 $\mathrm{M} \Omega$ electrode containing OGB- 1 solution $50-75$ $\mu \mathrm{m}$ below the surface of the slice. Gentle positive pressure was applied to the pipette, and OGB-1 was allowed to flow for 1.5-2 min per loading site. Loading was greatly enhanced at warm temperatures $\left(32-34^{\circ} \mathrm{C}\right)$ so most slices were loaded warm. Slices equilibrated for a minimum of 5 min after loading of the last OT site before imaging.

To drive Imc-projecting OT neurons antidromically, a bipolar electrode consisting of two $50-100 \mathrm{~K} \Omega$ tungsten electrodes with $\sim 400 \mu \mathrm{m}$ spacing was positioned across the dorsoventral extent of the Imc. Slices were bathed in a solution of $0.5-1 \mathrm{~mm}$ kynurenic acid to block glutamatergic transmission. Three trains of stimuli at $100-300 \mathrm{~Hz}$ were delivered to the Imc while imaging at $63 \times$ magnification. Epifluorescent illumination (EXFO) was passed through a filter cube (excitation, D450/50x; dichroic, 505DCXT; emission, E515LPv2; Chroma Technology). Illumination of tissue was controlled by a shutter (Smartshutter; Sutter Instruments). Images were captured for $6 \mathrm{~s}$ at a rate of 8 frames/s using Qcapture Pro 5.0 (QImaging). Movies were immediately analyzed to calculate $\Delta F / F$ with custom-made software in MATLAB (MathWorks). Putative neurons were differentiated from glia by the degree to which OGB-1 signals were temporally correlated with the onset and offset of antidromic stimulation (see Fig. $4 C$, bottom). To target recordings from antidromically activated neurons, an integrated poststimulus $\Delta F / F$ OGB-1 signal was computed and overlaid over the bright-field image (contrast enhanced using Dodt illumination) captured in the same plane.

After image capture, the kynurenic acid solution was washed off. Cells were targeted visually, and all putative Imc-projecting neurons were verified using trains of $100 \mathrm{~Hz}$ Imc stimulation. In cell-attached recordings, neurons that generated consistent, short-latency spikes with jitter of $<0.4 \mathrm{~ms}$ were deemed to be antidromically stimulated. In whole-cell recordings, the rise time of the spike also provided confirmation: antidromically stimulated spikes had no apparent PSP preceding spike initiation and were visible as unclamped spikes in voltage-clamp recordings. The position of the retinal afferent stimulating electrode was moved to a point on the pial surface that was radially aligned with the presumed direction of the apical dendrite and roughly at the same depth in the slice as that of the cell soma.

Non-antidromically activated neurons either (1) did not show definitive evidence of antidromic activation, as assayed by electrical recordings or (2) were chosen randomly from tissue that had been pressure injected with OGB-1.

\section{Anatomy}

Nissl-stained tissue (Fig. 1A,B) was prepared from $400 \mu \mathrm{m}$ acute slices fixed in $4 \%$ paraformaldehyde overnight. Slices were then washed in PBS, sunk in $30 \%$ sucrose, and resectioned to $50 \mu \mathrm{m}$ sections. Sections were incubated in PBS plus 0.1\% Triton X-100 (PBST) with 1:100 Neutravidin Oregon Green 488 (Invitrogen) and 1:100 Neurotrace 530/615 fluorescent Nissl stain (Invitrogen) for $1 \mathrm{~h}$ and then washed and coverslipped. Fluorescent images were taken on an epifluorescent microscope at $4 \times$ magnification.

For retrograde labeling of OT projections to the Imc (see Fig. 4A), 500 $\mu \mathrm{m}$ acute slices were prepared and placed into a static interface chamber containing ACSF bubbled with $95 \% \mathrm{O}_{2} / 5 \% \mathrm{CO}_{2}$. Biocytin (1\%) was dissolved in ACSF and pressure injected into the Imc. Slices were incubated for $>4 \mathrm{~h}$ and then fixed in $4 \%$ paraformaldehyde overnight. Slices were washed in PBS, sunk in 30\% sucrose, and resectioned to $50 \mu \mathrm{m}$ sections. Sections were placed into a solution of $0.1 \%$ $\mathrm{H}_{2} \mathrm{O}_{2}$ in PBST for $30 \mathrm{~min}$ and then washed three times with PBST. Sections were then placed in avidin-biotin solution (Vectastain, PK6100; Vector Laboratories) overnight at $4^{\circ} \mathrm{C}$. Slices were washed two times in PBST and then two times in Tris-buffered saline. 3,3'Diaminobenzidine (DAB kit, SK4100; Vector Laboratories) was made according to the kit protocol and applied for 5-10 min. Reactions were stopped with water, and the slices were mounted onto slides. After drying, slices were defatted and coverslipped. Bright-field images were taken at $4 \times$ and $20 \times$ magnification.

\section{Analyses}

All analyses were performed using custom-made software using MATLAB and automated so as to remain blind to cell type during analysis. Statistical comparisons were made using nonparametric tests (Wilcoxon's rank-sum test) and Bonferroni's corrected for multiple comparisons, as described in Results.

Evoked spike-rate analysis and spike rates from current injections. To calculate spike rates from both cell-attached and whole-cell recordings, traces were high-pass filtered (Butterworth) at $200 \mathrm{~Hz}$ and visually inspected to determine a threshold to count spikes. Spike rate after afferent stimulation was calculated over the first $200 \mathrm{~ms}$ after stimulus. Spike rate in response to current injections was calculated over the entire duration of the current injection (500 ms).

Calculation of evoked PSC rate and spectral content. Current traces were downsampled to $1 \mathrm{kHz}$ (see Fig. 2C,D). Then the derivative of the current signal was taken. A rate-of-rise cutoff was determined as $3 \times \mathrm{SD}$ of the differentiated signal; peaks were detected as negative excursions with a relative threshold of $173 \mathrm{pA} / \mathrm{s}$ (25th and 75th percentiles, 144 and 197 $\mathrm{pA} / \mathrm{s}$ ) with respect to the neighboring signal. Peaks were counted over a $200 \mathrm{~ms}$ poststimulus window. Spectra of PSC event times were computed using the Chronux toolbox in MATLAB, using the mtspecgrampt function bandpassed between 25 and $250 \mathrm{~Hz}$. PSC spectra from different neurons were normalized by dividing each spectrum by its maximum power (across the entire bandwidth).

Analysis of spontaneous EPSCs. A continuous trace of PSCs was recorded at a potential that minimized inward inhibitory currents (approximately $-55 \mathrm{mV}$, corrected for junction potential, just depolarized relative to the calculated reversal potential of $-61 \mathrm{mV}$ ); we could observe just-discernible outward IPSCs in the voltage-clamp trace. To determine the start times of putative EPSCs, a copy of the trace was downsampled to $1 \mathrm{kHz}$, high-pass filtered using a Butterworth filter with a high pass of 500 $\mathrm{Hz}$. Potential EPSCs were selected from the original, $10 \mathrm{kHz}$ sampled trace, aligned, and were included for analysis if they met statistically determined criteria for amplitude, rise time, and temporal separation from other PSCs.

Calculation of membrane time constant. Membrane voltage responses to a small hyperpolarizing current injection were recorded $\sim 20$ times, and the average trace was fit with a single exponential (Imc neurons) or double exponential (L10 neurons) using the Levenberg-Marquardt algorithm. The exponent that best matched the rate of membrane hyperpolarization was used to calculate the time constant.

Calculation of subthreshold membrane rectification. Membrane voltage responses to a series of hyperpolarizing and depolarizing current injections were analyzed $100 \mathrm{~ms}$ after initiation of the current injection, averaged over a $50 \mathrm{~ms}$ window. The change in membrane voltage $(\Delta V)$ between two sets of identically spaced current steps was calculated. The two sets of steps were (1) between the first two hyperpolarizing current steps relative to resting membrane potential ( $0 \mathrm{pA}$ injected) and (2) between the second and third depolarizing current steps relative to resting membrane potential. The rectification index was calculated as $\Delta V$ (depolarized) $/ \Delta V$ (hyperpolarized).

OGB-1 $\Delta \mathrm{F} / \mathrm{F}$ analysis. Movies were imported into MATLAB, and movie frames were registered to a recording of shutter opening, camera acquisition (frame start), and time of stimulus delivery. $\Delta F / F$ was calculated from an average baseline of the first three to four frames after shutter opening and before electrical stimulation. $\Delta F / F$ images were subjected to a 2-D median filter (medfilt2 in MATLAB) to smooth the signal over a $5 \times 5$ neighborhood.

\section{Calculation of spike half-width and after hyperpolarization parameters} For both L10 OT cell types, threshold traces (the trace corresponding to the first current injection that elicited action potentials, i.e., at rheobase) were used to calculate spike and afterhyperpolarization (AHP) parame- 

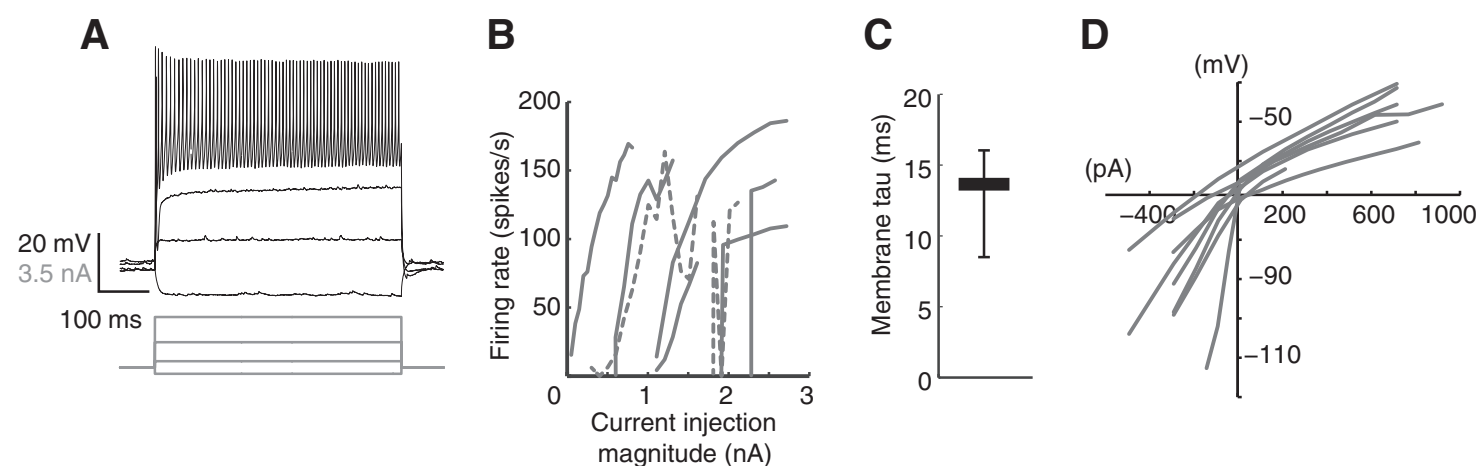

Figure 2. Membrane properties of Imc neurons. Bar plot conventions as in Figure 1E. A, Example of an Imc neuron recorded in current clamp firing at high rates in response to large current injections. Bottom gray lines indicate current injection. $\boldsymbol{B}$, Plots of firing rate as a function of injected current in $8 \mathrm{Imc}$ neurons. Neurons had varying rheobases and firing rate profiles in response to current injection. Across the population, peak rate was high. Solid and dotted lines are presented for visual clarity and have no meaning. C, Summary plot of Imc neuron membrane time constants. Median $\tau$ (25th and 75 th percentiles): $13.5 \mathrm{~ms}(8.5$ and $16.0 \mathrm{~ms}) ; n=11 . D$, Plots of subthreshold membrane potential as a function of injected current. Imc neurons show a clear reduction of $I-V$ slope (rectification) with depolarizing current injections $(n=8)$.

ters. AHP magnitude was determined as the difference between (1) the voltage at spike initiation (defined as the point at which the second derivative of the trace exceeded $10 \%$ of the maximum) and (2) the most hyperpolarized voltage in a $10 \mathrm{~ms}$ window after the spike. AHP duration was calculated as the time between the peak of the spike and that most hyperpolarized voltage within a $10 \mathrm{~ms}$ window after the spike.

Spike half-width was determined by finding the two points on the rising and falling phase $\geq 50 \%$ of the range of the voltages during the spike. The time between these two points was considered the half-width.

\section{Results}

An acute slice preparation of the midbrain maintains physiological characteristics of the Imc subnetwork

To study the subnetwork that controls the Imc, we required a preparation that allows precise targeting of the various midbrain network components. We reported previously that an acute slice preparation of the chick midbrain generates in vivo-like gammaband activity in the OT and Ipc in response to retinal afferent stimulation (Goddard et al., 2012). Furthermore, this preparation isolates the midbrain circuitry from all external sources of input, such as the retina and the forebrain. Therefore, any patterned activity observed in vitro is generated within the local midbrain circuitry preserved in the slice.

We verified that the high rates of firing observed in the Imc in vivo were present in this in vitro slice preparation. Acute transverse slices of the midbrain, $300 \mu \mathrm{m}$ thick, were prepared from 0 to 2-d-old chicks. At this age, these precocial birds are mostly self-sufficient; the midbrain visual system develops in ovo (LaVail and Cowan, 1971), and, within hours after hatching, animals are walking, feeding, and have sophisticated vision that allows imprinting on a parent (McCabe and Nicol, 1999), although additional visual system development continues to adulthood. We performed whole-cell patch-clamp recordings from Imc neurons and activated the midbrain network by electrically stimulating retinal afferents in the OT with a single $0.1 \mathrm{~ms}$ pulse (Fig. 1D). This stimulation evoked persistent barrages of spikes at high rates (median rate, 142 spikes/s; $n=19$ ) in Imc neurons (Fig. 1E). These rates of firing are somewhat lower than those recorded in vivo from the Imc in other avian species but are still far greater than the dominant rates of firing reported in the OT (Goddard et al., 2012). Thus, this preparation preserves, in part, the distinctive firing pattern of GABAergic Imc neurons.

\section{Intrinsic properties of Imc neurons}

We next set out to test the first hypothesis of how Imc neurons achieve their high firing rates. We first performed whole-cell, current-clamp recordings of Imc neurons and delivered somatic current injections. We observed that Imc neurons responded to step depolarizations with tonic, high discharge rates (Fig. $2 A$ ), in contrast to the bursting discharges exhibited by Ipc neurons (Goddard et al., 2012). Across the population of neurons, the peak rate had a median value was 145 spikes/s (25th and 75th percentiles, 117 and 166 spikes/s; $n=8)$. In some cases, Imc neurons fired fairly linearly in response to increasing current injection magnitude, although some responded nonlinearly by firing at high rates to currents just above threshold (Fig. 2B).

We next tested a crucial prediction of the first hypothesis: that Imc neurons should integrate inputs that arrive with low gamma periodicity (25-50 synaptic events/s, i.e., with interevent intervals of $20-40 \mathrm{~ms}$ ). This integration could occur if membrane time constants were longer than $\sim 30 \mathrm{~ms}$. The time constants of Imc neurons, in response to hyperpolarizing current injections, were shorter than the value required for integration: across a population of Imc neurons, membrane time constants had a median value of $13.5 \mathrm{~ms}$ (25th and 75th percentiles, 8.5 and $16.0 \mathrm{~ms}$; Fig. 2C).

Correspondingly, the membrane resistance of these neurons was low (median: $70 \mathrm{M} \Omega, 25^{\text {th }}, 75^{\text {th }}$ pct: $37.6,89.3 \mathrm{M} \Omega$ ), requiring large-magnitude current injections to achieve firing (median rheobase, $1108 \mathrm{pA}$; 25th and 75th percentiles, 453 and $1762 \mathrm{pA}$ ). Imc neurons also showed nonlinear subthreshold voltage responses to a series of hyperpolarizing and depolarizing current injections; the neurons showed clear rectification, with membrane responses to depolarizing current injections having a shallower slope than those in response to hyperpolarizing injections [median rectification index, $\Delta V$ (depolarized) $/ \Delta V$ (hyperpolarized), was $0.30 ; 25$ th and 75 th percentiles, 0.27 and $0.57 ; n=7$; Fig. $2 D]$. The observation of strong rectification suggests that the membrane time constant near threshold is even shorter, often by $>50 \%$, than the time constant estimated by the hyperpolarizing current injection. Thus, contrary to the expectations of the first hypothesis, the membrane properties of Imc neurons are specialized not for temporal integration.

\section{Synaptic inputs impinge on Imc neurons at high, not low, rates}

Because the membrane properties of Imc neurons do not permit the integration of periodic synaptic inputs, we next tested whether the properties of excitatory synapses would permit temporal integration. We recorded spontaneous EPSCs (sEPSCs) in Imc neurons using whole-cell voltage-clamp techniques. The time course of Imc synaptic currents was rapid: the median half- 
width of sEPSCs was $1.3 \mathrm{~ms}$ (25th and 75th percentiles, 1.2 and $1.7 ; n=15$; Fig. $3 A, B)$. These synaptic currents were also of large magnitude, with a median peak amplitude of $90.9 \mathrm{pA}$ (25th and 75th percentiles, 73.1 and 130.3 pA; Fig. 3B). Thus, the properties of individual synaptic events were also specialized to be rapid.

The short membrane time constants and rapid synaptic dynamics of Imc neurons suggest that, to achieve high rates of output, Imc neurons must receive high rates of input. To reveal the temporal structure of synaptic inputs to Imc neurons, we examined the pattern of evoked synaptic inputs using voltage-clamp recordings. We held neurons at $-55 \mathrm{mV}$ to isolate excitatory input and activated the OT with a $0.1 \mathrm{~ms}$, focal retinal afferent stimulation. This stimulation evoked barrages of rapid, large-magnitude EPSCs in Imc neurons (median EPSC rate, 113.5 $\mathrm{PSC} / \mathrm{s} ; 25$ th and 75 th percentiles, 92.1 and 136.5 PSC/s; $n=13$; Fig. $3 C$, bottom).

To assess whether this high rate of input correlated with the firing patterns in Imc neurons, we sequentially compared spike rates and PSC rates in a subset of neurons. Because of the recording conditions required for performing voltageclamp recordings in Imc neurons (see Materials and Methods), spike rates were recorded extracellularly before break-in, and subsequently, EPSCs were recorded in voltage clamp (Fig. 3C,D). We observed that both the spike rates and EPSC rates were high. Median spike rate was 133 spikes/s, and after break-in, EPSC rate was 126 events/s $(n=10)$. EPSC rates were estimated by finding large, rapid excursions in the recorded currents (see Materials and Methods). This approach provided a lower bound for the actual rates of EPSCs impinging on Imc neurons. However, this underestimation would suggest that true EPSC rates are even higher, unlike the periodic input into neighboring, cholinergic Ipc neurons (Goddard et al., 2012). In addition, spectral analysis of excitatory currents revealed broadband power, with no evidence of EPSC periodicity in the gamma-band range (Fig. $3 E$ ), unlike synaptic inputs to Ipc neurons (Goddard et al., 2012).

These results confirm that the persistent, high firing rates of Imc neurons are not a result of a transformation occurring in Imc neurons but instead reflect rapid barrages of excitatory input that are impinging on these neurons from the OT.

\section{Identification of Imc-projecting neurons using antidromically activated imaging-based mapping}

Next, we set out to test the remaining two hypotheses. These hypotheses can be discriminated by observing the firing rate of L10 neurons that project to the Imc. Hypothesis 2 predicts that Imc-projecting L10 neurons respond to afferent drive with low spike rates but that they fire with temporal jitter relative to each other. Alternatively, hypothesis 3 predicts that Imc-projecting L10 neurons respond to afferent drive with high rates. Neither type of neuron was reported in a previous, random sample of L10 neurons (Goddard et al., 2012). However, the proportion of Imcprojecting L10 neurons may be small (Fig. $4 A$ ) and, thus, not
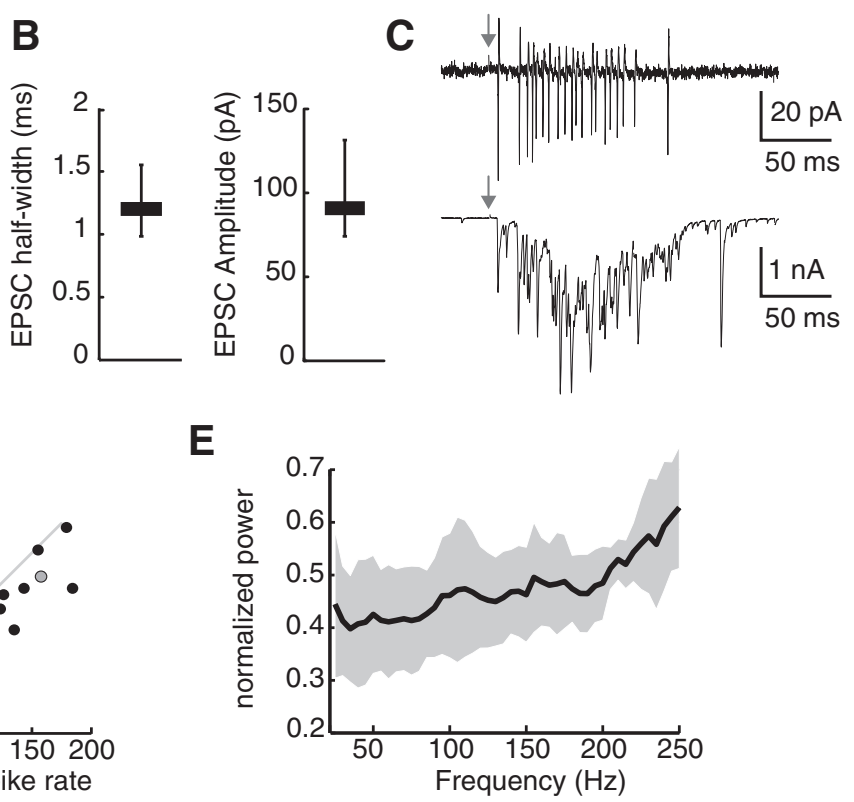

(spikes/s)

Figure 3. Synaptic properties of Imc neurons. $\boldsymbol{A}$, Averaged sEPSC from one Imc neuron showing rapid decay. $\boldsymbol{B}$, Left, Summary (Imc neurons. Median half-width (25th and 75th percentiles): $1.3 \mathrm{~ms}$ (1.2 and $1.7 \mathrm{~ms}$ ); $n=15$ neurons. (freque same neuron in voltage clamp (at $-55 \mathrm{mV}$ ). Gray arrow indicates time of ricrostimulation. D, Plot of spike rate versus EPSC rate for Imc neurons. Median spike rate (25th and 75t (133 spikes/s (125.5 and 152.8 spikes/s); EPSC rate: 126 PSC/s (115.5 and 150.2 PSC/s); $n=10$. Gray circle represents the neuron shown in $\boldsymbol{A}$. $\boldsymbol{E}$, Normalized point-process multitaper spectrum of detected PSC event times. Black line indicates mean

sampled previously. Therefore, we needed a method to identify the L10 OT neurons that project to the Imc.

We developed a technique to increase the likelihood of recording from neurons that send axons into the Imc (Fig. 4B). We combined axonal, antidromic stimulation in the Imc with the imaging of calcium-sensitive fluorescent dye in L10 neurons. We refer to this combined approach as antidromically activated imaging-based mapping (AIM). We bulk loaded neurons in L10 by focal pressure injection of the fluorescent calcium-sensitive dye OGB-1. The low-affinity glutamate receptor antagonist kynurenic acid (0.5-1 mM) was added to the bath to block synaptically mediated excitation. Next, a bipolar stimulating electrode was placed across the dorsoventral extent of the Imc (Fig. 4B).

Antidromic stimulation of the Imc induced calcium-related fluorescence signals in L10 neurons. Trains of stimuli (100-300 spikes/s) delivered to the Imc elicited reliable, stimulus-locked OGB-1 signals in the somata of a subset of L10 neurons imaged with epifluorescent microscopy (Fig. 4C). The resulting, baseline-subtracted OGB-1 signal was used to guide patch-clamp recordings of the putative antidromically activated neurons. Once recording from a putative Imc-projecting L10 neuron was established, we verified its antidromic activation by Imc microstimulation (Fig. $4 D$ ), as the kynurenic acid was washed out to permit activation of the OT by retinal afferent stimulation.

\section{Imc-projecting neurons, identified by AIM, fire at high rates}

Hypotheses 2 and 3 make distinct predictions for the temporal patterns of afferent-driven activity between two populations of L10 neurons (Fig. $1 C$ ): those that are antidromically activated by Imc microstimulation and those that are not antidromically activated. If both populations possess similar response rates to af- 


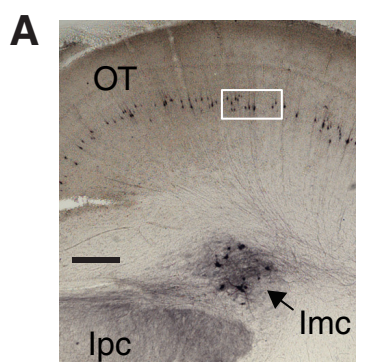

B
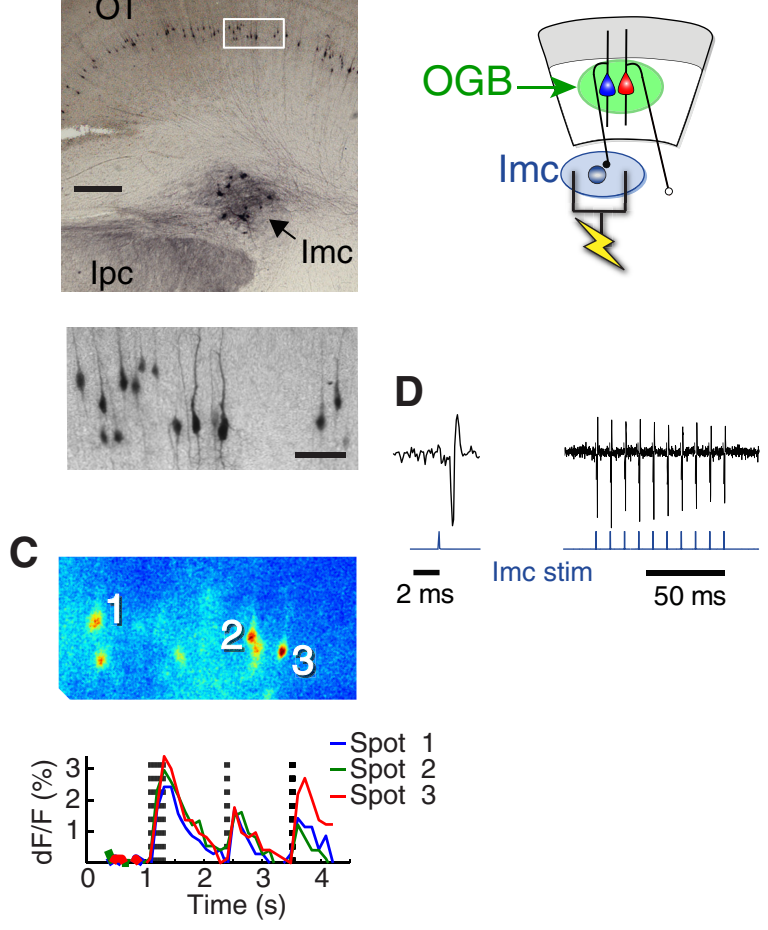

Figure 4. AIM. A, Top, Midbrain network after a biocytin injection placed in the Imc in an in vitro slice. Box indicates region shown in the bottom image. Scale bar, $250 \mu \mathrm{m}$. Bottom, Higherpower image of $\mathrm{L} 10$, showing retrogradely labeled neurons. Scale bar, $50 \mu \mathrm{m}$. B, Schematic of AIM experimental setup. OGB-1 was pressure injected into L10 in vitro. A bipolar electrode was placed across the Imc. The blue neuron represents an antidromically activated neuron, and the red neuron is not antidromically activated. C, Top, Image at $63 \times$ of $L 10$ showing the integrated $\Delta F / F$ OGB-1 signal after Imc stimulation. Red represents a large increase in fluorescence. Bottom, Time course of $\Delta F / F$ signal in three neurons indicated in the top image. Black, Dotted lines indicate times of antidromic stimulus trains. This image is not from the same tissue as shown in A. D, Left, A short-latency, extracellularly recorded L10 action potential (black) after a $0.1 \mathrm{~ms}$ electrical pulse (blue) delivered to the Imc. Right, A train of electrical stimuli delivered to the Imc at 100 spikes/s (bottom trace, blue) elicits a consistent, stimulus-locked train of L10 action potentials (top trace, black).

ferent stimulation, hypothesis 2 is more likely. In contrast, if antidromically activated neurons fire at higher rates than nonantidromically activated neurons, hypothesis 3 is supported. In support of hypothesis 3 , the two populations displayed dramatically different response rates to a focal, $0.1 \mathrm{~ms}$ retinal afferent stimulation (Fig. 5). Antidromically activated L10 neurons responded with persistent, high firing rates (median rate, 133 spikes/s; 25th and 75th percentiles, 90.5 and 203 spikes/s; $n=9$ ). In contrast, nonantidromically activated neurons responded with much lower firing rates (median rate, 48 spikes/s; 25th and 75th percentiles, 37.1 and 75.9 spikes/s; $n=17 ; p<0.01$, Wilcoxon's rank-sum test compared with antidromically activated neurons; Fig. $5 B, C$ ). These lower rates matched the rates reported previously for randomly sampled L10 neurons (Goddard et al., 2012).

The distinct rates described above raise a question: do these two populations of L10 OT neurons possess specialized biophysical properties for generating different firing rates in response to afferent stimulation? We addressed this question by testing L10 neurons with increasing steps of depolarizing current. We found that antidromically activated L10 neurons exhibited consistently higher maximal firing rates than non-antidromically activated neurons (Fig. $5 D-F$ ), as well as higher initial firing rates. Other firing properties between these two populations were also dis- tinct: antidromically activated neurons required larger currents to reach threshold (rheobase) and had narrower spike widths and shorter afterhyperpolarization durations (Fig. $5 G-J$ ). The magnitude of spike afterhyperpolarizations did not vary systematically between these two populations ( $p=0.62$; data not shown). These specializations in intrinsic excitability support the ability of antidromically activated neurons to respond to afferent drive with higher rates of firing. Antidromically activated neurons also had lower membrane resistances than non-antidromically activated neurons (Fig. 5K). Although time constants were nominally shorter in antidromically activated neurons, the two populations were not significantly different (median $\tau$ : antidromically activated, $8.1 \mathrm{~ms}$; non-antidromically activated, 15.0 ms; $p=0.01$, Wilcoxon's rank-sum test with Bonferroni's correction for multiple comparisons).

Overall, the biophysical specializations of antidromically activated L10 neurons allow them to transform an equivalent level of afferent drive into higher firing rates than the same drive onto neighboring non-antidromically activated neurons (Fig. 5E, dashed gray line). Thus, this subpopulation of L10 neurons is specialized to transform retinal input into high-rate discharges and to transmit this high-rate signal to the Imc. These relatively rare Imc-projecting L10 neurons are embedded within a much larger population of neurons that responds with lower rates and tends to synchronize at gamma frequencies with the LFP.

\section{Discussion}

The results presented here show that biophysical specializations in intercalated neural circuits perform a temporal transformation: they convert peripheral inputs into trains of spiking output, with spike rates aligned to the function of downstream subnetworks (Fig. 6). In this study, we reveal that OT L10 neurons that project to the GABAergic Imc temporally transform a brief stimulus into persistent, high-rate firing. This high firing rate contrasts sharply with the slower responses to the same stimulus exhibited by neighboring L10 OT neurons, such as those that project to the nearby cholinergic Ipc (Goddard et al., 2012). We also show that Imc neurons themselves are specialized to fire at high rates, have short time constants, and receive high-rate inputs. The architecture of Imc neurons, in turn, performs a spatial transformation: the axonal arbors of Imc neurons project broadly across the space maps in the OT and Ipc (Wang et al., 2004), delivering high-rate inhibitory activity that can powerfully suppress the representations of competing stimuli in the midbrain selection network (Fig. 6, left).

In the sections that follow, we discuss the novel technique we used to identify and study Imc-projecting L10 neurons in the OT, as well as the roles of high-rate firing and biophysical specializations in the transformation of information by neural circuits.

\section{AIM}

We developed AIM to identify neurons that project to specific targets in the brain. This technique combines imaging and electrical techniques to capitalize on two complementary strengths: high spatial and temporal resolution. The use of calcium indicators enables visual identification of neurons that have been antidromically activated by electrical microstimulation, an approach far more efficient than electrical recording techniques for locating sparsely distributed projection neurons. Once an antidromically activated neuron has been identified optically, whole-cell recording techniques can be applied to permit temporally precise and detailed analysis of cellular mechanisms that contribute to the coding properties of a neuron. 


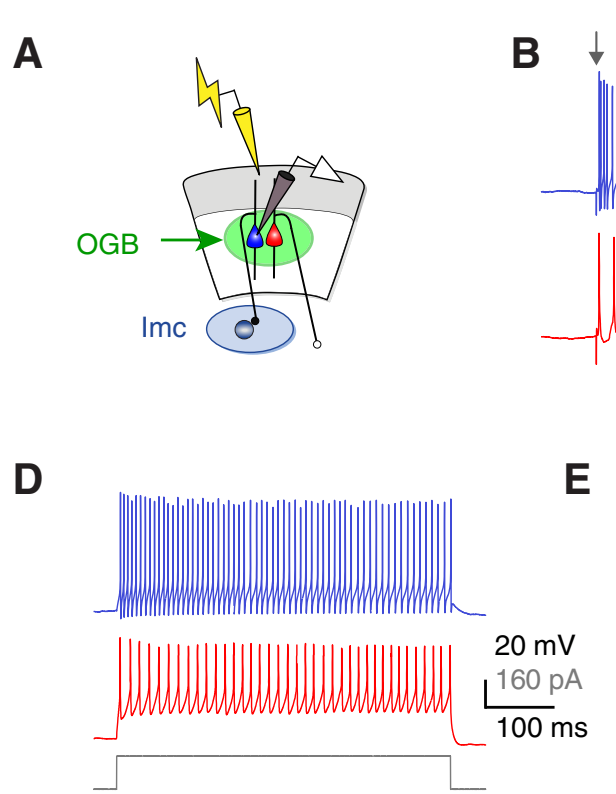

ant. activated non-ant. activated

E
G

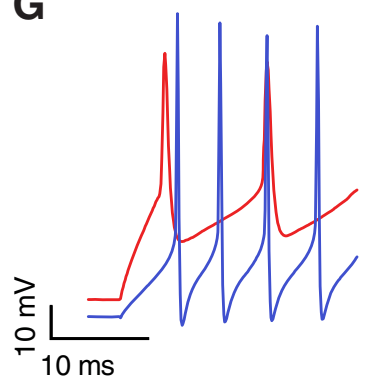

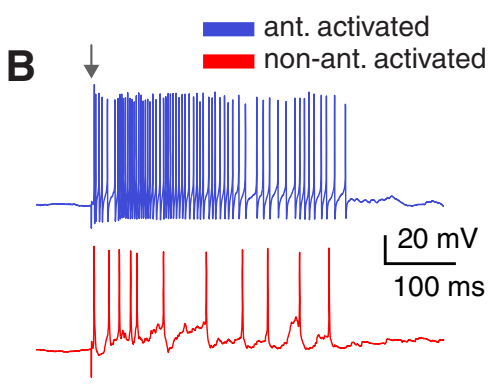

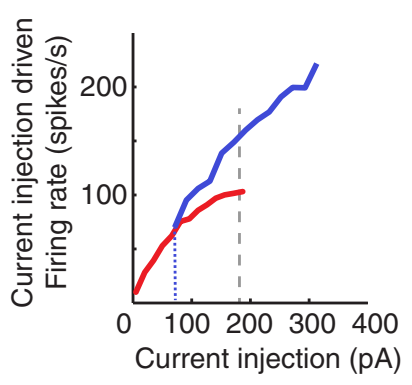

H

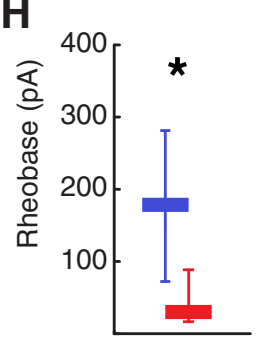

I

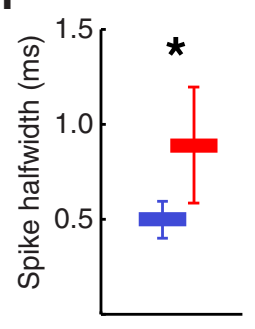

J

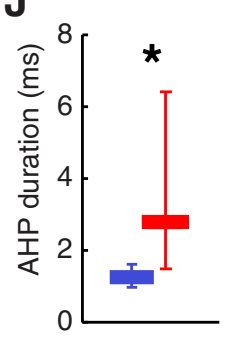

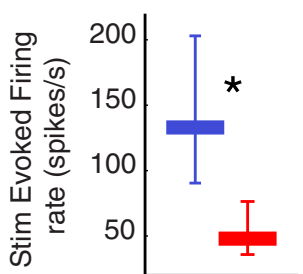

F

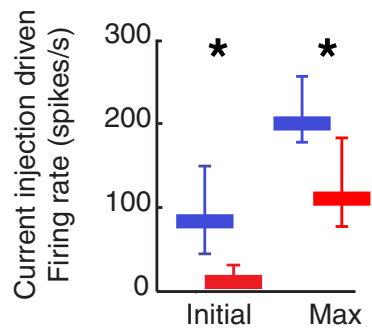

K

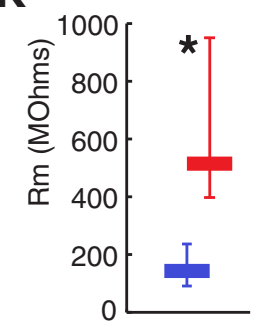

Figure 5. AIM uncovers high firing rate neurons in L10 that project to the Imc with distinctive physiological properties. Bar plot conventions as in Figure $1 E$. A, Schematic of experimental setup. Same as in Figure $4 B$ but with stimulating electrode placed at the retinal afferent layer. $B$, An antidromically (ant.) activated, putative Imc-projecting L10 neuron (blue; same as in Fig. 3D) fires at a higher rate than a non-antidromically (non-ant.) activated neuron (red) in response to $0.1 \mathrm{~ms}$ retinal afferent stimulation (gray arrow). $C$, Summary plot. In response to retinal afferent stimulation, antidromically activated L10 neurons (blue) responded with a high median (25th and 75th percentiles) spike rate: 133 spikes $/ \mathrm{s}$ (90.5 and 203 spikes/s); $n=9$. In contrast, non-antidromically activated neurons (red) responded with a lower spike rate: 48 spikes $/ \mathrm{s}$ ( 37.1 and $75.9 \mathrm{spikes} / \mathrm{s}$ ); $n=17 ; p<0.01$. $\boldsymbol{D}$, In response to identical step current injections (gray line), firing rate in an antidromically activated neuron (blue) is higher than in a non-antidromically activated neuron (red). Same neurons as shown in Figure 5B. $E$, Plot of firing rate versus current injected for the two neurons shown in $\boldsymbol{D}$. Antidromically activated neurons (blue) fire at higher rates than non-antidromically activated (red) across various magnitudes of current injection. Gray line represents current injection magnitude shown in $\boldsymbol{D}$. $\boldsymbol{F}$, Summary plot of firing rates: antidromically activated L10 neurons (blue) show higher initial (left) and maximal (right) firing rates than non-antidromically activated neurons (red). Median initial rate in antidromically activated neurons: 84.6 spikes $/ \mathrm{s}$ (20.9 and 147.0 spikes $/ \mathrm{s}), n=14$; non-antidromically activated: $13.2 \mathrm{spikes} / \mathrm{s}$ ( 8.4 and 31.4 spikes/s); $n=33 ; p<0.009$. Median maximal firing rate (25th and 75th percentiles) in antidromically activated neurons: 201.4 spikes $/ \mathrm{s}$ (175.5 and 256.4 spikes $/ \mathrm{s}$ ), $n=14 ;$ non-antidromically activated: 110.6 spikes/s (77.3 and 182.7 spikes/s), $n=33 ; p<0.001$, Wilcoxon's rank-sum test. $\mathbf{G}$, Neurons shown in Figure $6 B$ at expanded timescale in response to an $\sim 50 \mathrm{pA}$ injection. $\boldsymbol{H}$, Summary plot of rheobase: antidromically activated $L 10$ neurons (blue) require a higher current magnitude to reach threshold than non-antidromically activated neurons (red). Median rheobase in antidromically activated neurons: $175 \mathrm{pA}(76$ and $277 \mathrm{pA}), n=14$; non-antidromically activated: $40 \mathrm{pA}$ (20 and $76 \mathrm{pA}), n=33 ; p<0.002$, Wilcoxon's rank-sum test. I, Summary plot of spike half-width: antidromically activated L10 neurons (blue) have narrower spike half-widths than non-antidromically activated neurons (red). Median spike half-width in antidromically activated neurons: $0.5 \mathrm{~ms}$ ( 0.4 and $0.6 \mathrm{~ms}), n=14$; non-antidromically activated: $0.9 \mathrm{~ms}(0.6$ and $1.2 \mathrm{~ms}), n=33 ; p<0.002$, Wilcoxon's rank-sum test.J, Summary plot of AHP duration:antidromically activated L10 neurons (blue) have shorter AHPs than non-antidromically activated neurons (red). Median AHP duration in antidromically activated neurons: $1.2 \mathrm{~ms}$ ( 0.9 and $1.6 \mathrm{~ms}), n=14$; non-antidromically activated: $2.8 \mathrm{~ms}(1.5$ and $6.4 \mathrm{~ms}$ ), $n=33 ; p<0.002$, Wilcoxon's rank-sum test. $\boldsymbol{K}$, Summary plot of membrane resistance: antidromically activated L10 neurons (blue) have lower: $139 \mathrm{M} \Omega$ ( 93.5 and 229.3 $\mathrm{M} \Omega), n=14$; non-antidromically activated: $591.3 \mathrm{M} \Omega(311.7$ and $1003.4 \mathrm{M} \Omega), n=33 ; p<0.001$, Wilcoxon's rank-sum test.

AIM enables the dissection of microcircuits with high spatial and temporal resolution both in vivo and in vitro and in genetically intractable species. AIM offers several important advantages over standard virus or fluorophore injections. (1) For online modulation, antidromic electrical stimulation allows for online adjustments of stimulation parameters for precise targeting of the electrode and thus the activation of specific populations of projection neurons. In contrast, large injection volumes or mistargeting of dye or virus can label unwanted populations of neurons. (2) For specificity, many target structures, particularly deep in the brain, are difficult to locate. If targets exhibit particular physiological signatures, such as selectivity to a certain stimulus modality or intrinsic spontaneous firing patterns, AIM can exploit these physiological signatures to position the stimulating electrode before stimulation. (3) For flexibility/adaptability, AIM enables the identification of multiple projection targets of a given circuit. One can place multiple stimulating electrodes in different target regions or move a single stimulating electrode from one region to another. For a comparable experiment using fluorescent indicators, the limitations in spectral separation among different fluo- 


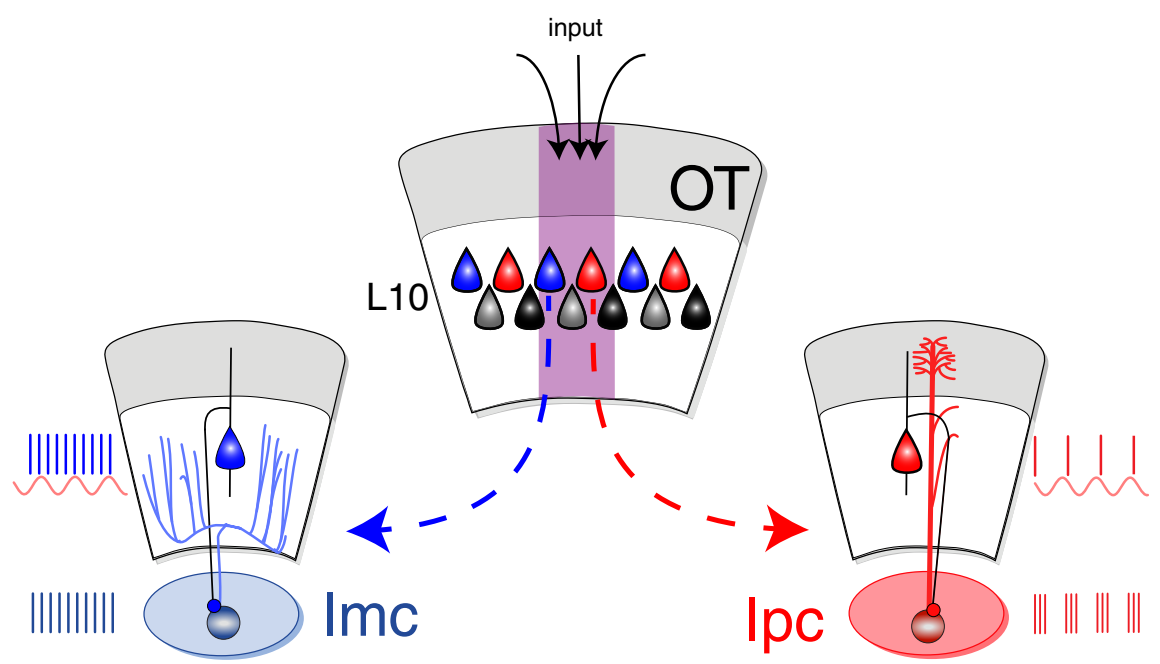

Figure 6. Summary schematic of parallel microcircuits in the 0T. Several subtypes of L10 neurons in the 0T, shown in different colors, form parallel microcircuits that tile space. For each location in space (purple shading), a set of microcircuits receives topographic input and projects to different downstream targets. Left, One 0T microcircuit transforms afferent input into high firing rate output. This output goes to the Imc, which broadcasts high rates of inhibition across the OT and Ipc space maps. Right, Another OT microcircuit transforms afferent input into periodic, lower firing rate output. This output goes to the Ipc, which delivers periodic bursts of activity focally to one place in the OT space map (Goddard et al., 2012).

rophores fundamentally limit the number of populations that can be tested simultaneously. (4) For timing, AIM can be applied rapidly within the course of a single experimental session. In contrast, dye or virus injections place time constraints on experiments: dyes may transport quickly but may fade with time; genetic manipulations, involving viruses or electroporation, often require long periods of time to achieve sufficient protein expression.

Various calcium indicators can be used for AIM. The use of injectable, membrane-permeable calcium indicators, such as OGB-1, allows for imaging of structures in genetic intractable species. However, genetically encoded calcium indicators provide great advantages for studying populations over time and do not require pressure injection of a DMSO-containing solution. In our slice preparation, we added synaptic blockers to the imaged region to eliminate stimulation-evoked afferent drive. In an in vivo preparation, brief, local perfusion of synaptic blockers may be required to eliminate unwanted fluorescent signals that result from afferent synaptic activation. Synaptic blockade has the additional benefit of increasing signal-to-noise by reducing background activity. Thus, this approach enables efficient identification of microcircuits in intact and semi-intact preparations across a wide range of species.

\section{Gating inhibitory circuits are characterized by high firing rates}

Inhibitory circuits that gate information tend to fire at conspicuously high rates. High rates presumably cause these circuits to release a continuous stream of GABA and to potently suppress their downstream targets. For example, the basal ganglia play an important role in gating motor output (Hikosaka et al., 2000; Seger, 2008), and inhibitory output neurons of the basal ganglia fire at rates of 50-150 spikes/s (Hikosaka and Wurtz, 1983; Handel and Glimcher, 2000; Goldberg and Fee, 2012). Similarly, the reticular nucleus of the thalamus may play an important role in gating sensory information into the cortex (Crick, 1984; McAlonan et al., 2008), and inhibitory output neurons of the reticular nucleus can fire at rates in excess of 500 spikes/s (Pinault, 2004;
McAlonan et al., 2008). Some gating inhibitory neurons have high spontaneous rates, whereas others fire at high rates in response to afferent drive. In either case, the high rate of inhibitory neuron firing can play a crucial role in gating and selecting certain representations.

The Imc plays an analogous role in gating sensory information in the midbrain selection network (Marín et al., 2007; Mysore and Knudsen, 2013). Imc neurons fire at exceptionally high rates, with some studies reporting rates above 500 spikes/s (Marín et al., 2007). The high firing rates of Imc neurons enable them to suppress the representations of strong, competing stimuli in the OT and Ipc.

In this study, we identify a new component of the Imc subnetwork: the excitatory L10 OT neurons that project to the Imc. These sparsely distributed L10 neurons are distinguished by their high firing rates. The GABAergic, inhibitory neurons in the Imc respond to this excitatory drive with similarly high firing rates as well. Thus, although these Imc-projecting L10 neurons are themselves excitatory, they directly drive Imc activity and, therefore, are essential to the inhibitory gating mechanism. This principle may extend to other networks in the brain: neurons that are themselves excitatory may be primarily involved in driving inhibitory and other modulatory neurons and may control the timing and spatial pattern of inhibitory gates and neuromodulatory tone.

\section{Functional roles of biophysical specializations}

Neighboring neurons within a brain structure possess distinct biophysical and synaptic specializations. These specializations may be crucial for the generation of distinct patterns of discharge. Such specializations are often associated with neurons that have different downstream targets (Kasper et al., 1994; Dutar et al., 1998; Brown and Hestrin, 2009; Angelo et al., 2012). For example, cortical neurons that project to the superior colliculus have distinctive bursting properties and short membrane time constants compared with those that project callosally (Kasper et al., 1994). In the nucleus HVC (higher vocal center) in songbirds, three subtypes of neurons project to different target nuclei, and each subtype has distinctive physiological properties, including spike width, AHP timing and kinetics, and sensitivity to glutamate and GABA (Dutar et al., 1998). In addition, mitral cells in the rodent olfactory bulb that are associated with a single glomerular circuit show similar magnitudes of hyperpolarization-activated depolarizing currents ("h-current"), whereas those in different glomerular circuits do not (Angelo et al., 2012).

We found analogous biophysical specializations within microcircuits in the avian stimulus selection network. Imcprojecting L10 OT neurons possess a different constellation of biophysical specializations than do neighboring L10 neurons. These specializations imbue neighboring neurons with different membrane properties to generate distinct firing patterns in response to equivalent afferent input (Figs. 5, 6). Specializations may include differential expression of potassium channels, which are known to influence membrane resistance (Hu et al., 2010) and properties of AHP (Storm, 1987; Zhang and McBain, 1995). In addition, the synaptic inputs on L10 OT neurons are also likely 
to be specialized: because Imc-projecting neurons possess lower membrane resistances (Fig. $5 K$ ), larger synaptic conductances would be required to depolarize this population of neurons. This prediction is supported by the observation that the current required to fire action potentials is greater among Imc-projecting L10 OT neurons than among non-Imc-projecting L10 neurons (Fig. 5H). Additional studies are needed to dissect the cellular and network mechanisms that control the persistent synaptic drive impinging on L10 neurons.

The biophysical specializations of Imc-projecting L10 neurons have a clear functional role: to generate high rates of discharge. This high rate, when passed to the Imc, permits the constant release of GABA from the Imc needed to suppress competing stimulus representations in the network. The other populations of L10 OT neurons exhibit different biophysical specializations for transforming afferent input. L10 neurons that project to the cholinergic Ipc fire periodically, causing Ipc neurons to burst with gamma periodicity. This periodic bursting leads to the enhancement of sensory representations in the OT and is crucial for signal transfer to higher-order forebrain areas (Marín et al., 2007). Thus, distinctive temporal transformations of afferent drive by neighboring microcircuits in L10 are crucial to the respective functions of their downstream subnetworks.

\section{References}

Angelo K, Rancz EA, Pimentel D, Hundahl C, Hannibal J, Fleischmann A, Pichler B, Margrie TW (2012) A biophysical signature of network affiliation and sensory processing in mitral cells. Nature 488:375-378. CrossRef Medline

Armstrong KM, Fitzgerald JK, Moore T (2006) Changes in visual receptive fields with microstimulation of frontal cortex. Neuron 50:791-798. CrossRef Medline

Brown SP, Hestrin S (2009) Intracortical circuits of pyramidal neurons reflect their long-range axonal targets. Nature 457:1133-1136. CrossRef Medline

Crick F (1984) Function of the thalamic reticular complex: the searchlight hypothesis. Proc Natl Acad Sci U S A 81:4586-4590. CrossRef Medline

Dutar P, Vu HM, Perkel DJ (1998) Multiple cell types distinguished by physiological, pharmacological, and anatomic properties in nucleus $\mathrm{HVc}$ of the adult zebra finch. J Neurophysiol 80:1828-1838. Medline

Gazzaley A, Cooney JW, Rissman J, D’Esposito M (2005) Top-down suppression deficit underlies working memory impairment in normal aging. Nat Neurosci 8:1298-1300. CrossRef Medline

Goddard CA, Sridharan D, Huguenard JR, Knudsen EI (2012) Gamma oscillations are generated locally in an attention-related midbrain network. Neuron 73:567-580. CrossRef Medline

Goldberg JH, Fee MS (2012) A cortical motor nucleus drives the basal ganglia-recipient thalamus in singing birds. Nat Neurosci 15:620-627. CrossRef Medline

Handel A, Glimcher PW (2000) Contextual modulation of substantia nigra pars reticulata neurons. J Neurophysiol 83:3042-3048. Medline

Herrero JL, Roberts MJ, Delicato LS, Gieselmann MA, Dayan P, Thiele A
(2008) Acetylcholine contributes through muscarinic receptors to attentional modulation in V1. Nature 454:1110-1114. CrossRef Medline

Hikosaka O, Wurtz RH (1983) Visual and oculomotor functions of monkey substantia nigra pars reticulata. II. Visual responses related to fixation of gaze. J Neurophysiol 49:1254-1267. Medline

Hikosaka O, Takikawa Y, Kawagoe R (2000) Role of the basal ganglia in the control of purposive saccadic eye movements. Physiol Rev 80:953-978. Medline

Hu H, Martina M, Jonas P (2010) Dendritic mechanisms underlying rapid synaptic activation of fast-spiking hippocampal interneurons. Science 327:52-58. CrossRef Medline

Kasper EM, Larkman AU, Lübke J, Blakemore C (1994) Pyramidal neurons in layer 5 of the rat visual cortex. I. Correlation among cell morphology, intrinsic electrophysiological properties, and axon targets. J Comp Neurol 339:459-474. CrossRef Medline

Knudsen EI (2011) Control from below: the role of a midbrain network in spatial attention. Eur J Neurosci 33:1961-1972. CrossRef Medline

LaVail JH, Cowan WM (1971) The development of the chick optic tectum. I. Normal morphology and cytoarchitectonic development. Brain Res 28:391-419. CrossRef Medline

Lovejoy LP, Krauzlis RJ (2010) Inactivation of primate superior colliculus impairs covert selection of signals for perceptual judgments. Nat Neurosci 13:261-266. CrossRef Medline

Marín G, Salas C, Sentis E, Rojas X, Letelier JC, Mpodozis J (2007) A cholinergic gating mechanism controlled by competitive interactions in the optic tectum of the pigeon. J Neurosci 27:8112-8121. CrossRef Medline

McAlonan K, Cavanaugh J, Wurtz RH (2008) Guarding the gateway to cortex with attention in visual thalamus. Nature 456:391-394. CrossRef Medline

McCabe BJ, Nicol AU (1999) The recognition memory of imprinting: biochemistry and electrophysiology. Behav Brain Res 98:253-260. CrossRef Medline

Miller EK, Buschman TJ (2013) Cortical circuits for the control of attention. Curr Opin Neurobiol 23:216-222. CrossRef Medline

Mysore SP, Knudsen EI (2013) A shared inhibitory circuit for both exogenous and endogenous control of stimulus selection. Nat Neurosci 16:473478. CrossRef Medline

Pinault D (2004) The thalamic reticular nucleus: structure, function and concept. Brain Res Brain Res Rev 46:1-31. CrossRef Medline

Seger CA (2008) How do the basal ganglia contribute to categorization? Their roles in generalization, response selection, and learning via feedback. Neurosci Biobehav Rev 32:265-278. CrossRef Medline

Sridharan D, Boahen K, Knudsen EI (2011) Space coding by gamma oscillations in the barn owl optic tectum. J Neurophysiol 105:2005-2017. CrossRef Medline

Storm JF (1987) Action potential repolarization and a fast afterhyperpolarization in rat hippocampal pyramidal cells. J Physiol 385:733759. Medline

Wang Y, Major DE, Karten HJ (2004) Morphology and connections of nucleus isthmi pars magnocellularis in chicks (Gallus gallus). J Comp Neurol 469:275-297. CrossRef Medline

Zhang L, McBain CJ (1995) Potassium conductances underlying repolarization and after-hyperpolarization in rat CA1 hippocampal interneurones. J Physiol 488:661-672. Medline 